\title{
Correction: Reaching Those At Risk for Psychiatric Disorders and Suicidal Ideation: Facebook Advertisements to Recruit Military Veterans
}

Alan R Teo ${ }^{1,2,3}$, MD, MS; Samuel BL Liebow ${ }^{2}$, BA; Benjamin Chan ${ }^{3}$, MS; Steven K Dobscha ${ }^{1,2}$, MD; Amanda L Graham $^{4,5}, \mathrm{PhD}$

${ }^{1}$ HSR\&D Center to Improve Veteran Involvement in Care (CIVIC), VA Portland Health Care System, Portland, OR, United States

${ }^{2}$ Department of Psychiatry, Oregon Health \& Science University, Portland, OR, United States

${ }^{3}$ School of Public Health, Oregon Health \& Science University and Portland State University, Portland, OR, United States

${ }^{4}$ Schroeder Institute for Tobacco Research \& Policy Studies, Truth Initiative, Washington, DC, United States

${ }^{5}$ Department of Oncology, Georgetown University Medical Center, Washington, DC, United States

\section{Corresponding Author:}

Alan R Teo, MD, MS

HSR\&D Center to Improve Veteran Involvement in Care (CIVIC)

VA Portland Health Care System

3710 SW US Veterans Hospital Road (R\&D 66)

Portland, OR, 97239-2964

United States

Phone: 15032208262 ext 52461

Email: teoa@ohsu.edu

\section{Related Article:}

Correction of: http://mental.jmir.org/2018/3/e10078/

(JMIR Ment Health 2019;6(1):e13035) doi: 10.2196/13035

The authors of "Researching Those At Risk for Psychiatric Disorders and Suicidal Ideation: Facebook Advertisements to Recruit Military Veterans" (JMIR Ment Health 2018;5(3):e10078) incorrectly labeled some column headers in Table 2. Currently, the columns list the click-through rate as a percentage ("CTR", n (\%)"). They should be labeled as simply the number and click-through rate ("Number $\left(\mathrm{CTR}^{\mathrm{a}}\right)$ ").
The correction will appear in the online version of the paper on the JMIR website on January 9, 2019, together with the publication of this correction notice. Because this was made after submission to PubMed, PubMed Central, and other full-text repositories, the corrected article also has been resubmitted to those repositories.

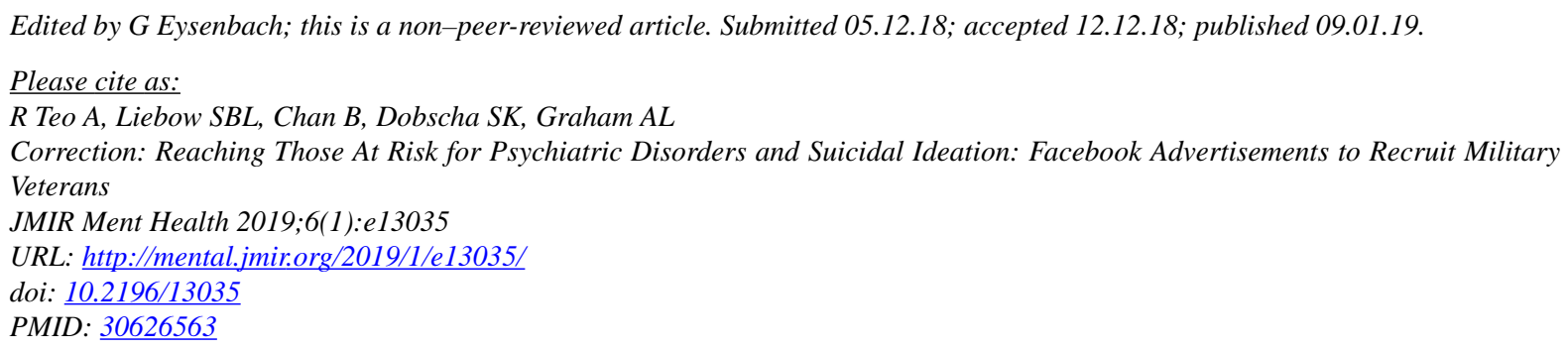

(C)Alan R Teo, Samuel BL Liebow, Benjamin Chan, Steven K Dobscha, Amanda L Graham. Originally published in JMIR Mental Health (http://mental.jmir.org), 09.01.2019. This is an open-access article distributed under the terms of the Creative Commons Attribution License (https://creativecommons.org/licenses/by/4.0/), which permits unrestricted use, distribution, and reproduction in any medium, provided the original work, first published in JMIR Mental Health, is properly cited. The complete bibliographic 
information, a link to the original publication on http://mental.jmir.org/, as well as this copyright and license information must be included. 